\title{
The Minimum Energy Principle in Description of Nonlinear Properties of Orthotropic Material
}

\author{
Tadeusz Wegner, Dariusz Kurpisz \\ Applied Mechanics Institute, Poznan University of Technology, Poznan, Poland \\ Email: tadeusz.wegner@put.poznan.pl, dariusz.kurpisz@put.poznan.pl
}

Received 2012

\begin{abstract}
In this paper the conception of theoretical determine the relations between material experimental characteristics is presented. On the base of stress-strain relations for nonlinear elastic anisotropic material and geometrical interpretation of deformation state, the general form of strain energy density function was introduced. Using this function and variational methods the relations between material characteristics were achieved. All considerations are illustrated by a short theoretical example.
\end{abstract}

Keywords: Material Characteristics; Mechanical Properties; Deformation State Components; Strain Energy Density Function; Minimum Energy Principle; Variational Methods

\section{Introduction}

One of the most important nature laws is the minimum energy principle. Thought of this principle each physical match tends at minimization of its energetic state. So in the case of material the configuration of deformation state must satisfy the principle of minimum of energy. Because the discussed law introduces relations between deformation state components, the material characteristics must be mutually coherent. On important rule of energy, as a tool to description of material mechanical properties, call attention Ogden [1], Perzyna [2,3], Petryk [4-6], Schroder [7], Wagner [8], Wegner [9] and other. The main aim of this paper is construction mathematical relations between material mechanical characteristics due to minimum of energy principle. For affirmation of generality of considerations the nonlinear elastic orthotropic material will be used.

\section{Geometrical Interpretation of Deformation State and Strain Energy Density Function}

The deformation of material requires the work of external load. Direct answer of material for application of external load program is the stress. Its value is dependent on load magnitude, deformation state and individual properties of material. So the relations between stress and strain (there be measure of deformation) are different for different materials. Connecting factors of strain and stress components are material characteristics. These relations for nonlinear elastic orthotropic material can be written as

$$
\left\{\begin{array}{l}
\varepsilon_{1}=\frac{\sigma_{1}}{E_{1}\left(\varepsilon_{1}\right)}-v_{12}\left(\varepsilon_{2}\right) \frac{\sigma_{2}}{E_{2}\left(\varepsilon_{2}\right)}-v_{13}\left(\varepsilon_{3}\right) \frac{\sigma_{3}}{E_{3}\left(\varepsilon_{3}\right)}, \\
\varepsilon_{2}=-v_{21}\left(\varepsilon_{1}\right) \frac{\sigma_{1}}{E_{1}\left(\varepsilon_{1}\right)}+\frac{\sigma_{2}}{E_{2}\left(\varepsilon_{2}\right)}-v_{23}\left(\varepsilon_{3}\right) \frac{\sigma_{3}}{E_{3}\left(\varepsilon_{3}\right)}, \\
\varepsilon_{3}=-v_{31}\left(\varepsilon_{1}\right) \frac{\sigma_{1}}{E_{1}\left(\varepsilon_{1}\right)}-v_{32}\left(\varepsilon_{2}\right) \frac{\sigma_{2}}{E_{2}\left(\varepsilon_{2}\right)}+\frac{\sigma_{3}}{E_{3}\left(\varepsilon_{3}\right)},
\end{array}\right.
$$

where $\sigma_{1}, \sigma_{2}, \sigma_{3}$ and $\varepsilon_{1}, \varepsilon_{2}, \varepsilon_{3}$ are respectively principal stress and strain components. $E_{i}\left(\varepsilon_{i}\right) \stackrel{\text { def. }}{=} \sigma_{i}\left(\varepsilon_{i}\right) / \varepsilon_{i}$ for $i=1,2,3$ and $v_{i j}\left(\varepsilon_{j}\right)=\varepsilon_{i} / \varepsilon_{j}$ for $i, j=1,2,3$ and $i \neq j$ are experimental material characteristics obtained in uniaxial tension tests. As we can see the full description of material requires an experimental assignment of nine characteristics. There is oppressive for realization. Hence it is proper to search for dependences among them.

\subsection{Geometrical Interpretation of Deformation State}

Let's assume that the external load program is such, that the orthotropy directions and principal deformation directions are the same. Next separate the material piece in shape a cube, that edges are parallel to principal orthotropy directions. The deformation process of this elementary cube was illustrated below.

Every deformation state response a point on deformation path C. On the end of this path we have a desired deformation state $\varepsilon_{1}{ }^{K}, \varepsilon_{2}{ }^{K}, \varepsilon_{3}{ }^{K}$. The motion along path $\mathrm{C}$ is initiated by changeable stress components. So every displacement along path C needs work.

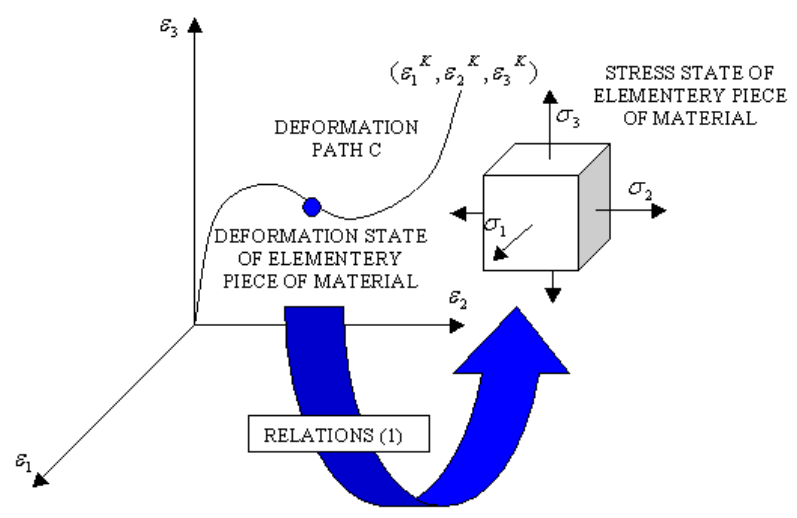

Figure 1. Interpretation of deformation state. 


\subsection{Strain Energy Density Function}

The deformation work $L$ can be expressed by the use of line integral in the form

$$
L=\int_{C} \sum_{i=1}^{3} \sigma_{i} d \varepsilon_{i}
$$

Because the space of deformation components is potential the deformation work is independent from the shape of deformation path $\mathrm{C}$. So we can write

$$
C=\left\{\begin{array}{l}
\varepsilon_{1}=\varepsilon_{1}{ }^{K} t, \\
\varepsilon_{2}=\varepsilon_{2}{ }^{K} t, \quad \text { where } t \in\langle 0,1\rangle \\
\varepsilon_{3}=\varepsilon_{3}{ }^{K} t .
\end{array}\right.
$$

and hence by the use of (2)

$$
W\left(\varepsilon_{1}{ }^{K}, \varepsilon_{2}{ }^{K}, \varepsilon_{3}{ }^{K}\right)=\int_{0}^{1} \sum_{i=1}^{3} \sigma_{i}\left(\varepsilon_{1}{ }^{K} t, \varepsilon_{2}{ }^{K} t, \varepsilon_{3}{ }^{K} t\right) \varepsilon_{i}{ }^{K} d t
$$

where $\sigma_{i}\left(\varepsilon_{1}, \varepsilon_{2}, \varepsilon_{3}\right)$ is the solution of system of Equations (1). The strain energy $W$ is a function of deformation state components.

\section{The Principle of Minimum Energy}

Because the solution of system of Equations (1) can be write in the form

$$
\sigma_{i}=E_{i}\left(\varepsilon_{i}\right) \cdot F_{i}\left(v_{12}, \ldots, v_{32}\right)
$$

for $i=1,2,3$, then the equation (4) can be written as

$$
\begin{aligned}
& W\left(\varepsilon_{1}{ }^{K}, \varepsilon_{2}{ }^{K}, \varepsilon_{3}{ }^{K}\right) \\
= & \int_{0}^{1} \sum_{i=1}^{3} E_{i}\left(\varepsilon_{i}{ }^{K} t\right) F_{i}\left(v_{12}\left(\varepsilon_{2}{ }^{K} t\right), \ldots, v_{32}\left(\varepsilon_{2}{ }^{K} t\right)\right) \varepsilon_{i}{ }^{K} d t .
\end{aligned}
$$

The right side of equation (6) is a functional due to functions $E_{i}\left(\varepsilon_{i}\right)$ and $v_{i j}\left(\varepsilon_{j}\right)$. Hence the detection of minimum of energy is equivalent the determination of minimum of functional (6). Let's put that

$$
\begin{aligned}
& F\left(E_{1}, E_{2}, E_{3}, v_{12}, \ldots, v_{32}\right) \\
= & \sum_{i=1}^{3} E_{i}\left(\varepsilon_{i}{ }^{K} t\right) F_{i}\left(v_{12}\left(\varepsilon_{2}{ }^{K} t\right), \ldots, v_{32}\left(\varepsilon_{2}{ }^{K} t\right)\right) \varepsilon_{i}{ }^{K} .
\end{aligned}
$$

If (6) has a minimum, then the following assumptions must be satisfied

$$
\begin{gathered}
\text { (I-III) } \underset{1 \leq i \leq 3}{\forall} \frac{\partial F}{\partial E_{i}}=0 \\
(\mathrm{IV}-\mathrm{IX}) \frac{\partial F}{\partial v_{i j}}=0 \text { for } i, j=1,2,3 \text {, and } i \neq j .
\end{gathered}
$$

The Equations (8) and (9) introduce (on the base of minimum of energy), the relations between material mechanical characteristics. So if single or twice indexed material characteristics are known then the determination of the next characteristics is possible.

\section{An Example}

Let's assume, that we have nonlinear elastic isotropic material under flat state of stress (in plane 1O2). In such case the ma- terial physical properties are the same in all directions. It means that there are two material characteristics. Because the component of stress state in third direction is equal zero $\left(\sigma_{3}=0\right)$, the deformation path $\mathrm{C}$ can be given as

$$
C:\left\{\begin{array}{l}
\varepsilon_{1}=\varepsilon_{1}{ }^{K} t \\
\varepsilon_{2}=\varepsilon_{2}{ }^{K} t
\end{array} \text { for } t \in\langle 0,1\rangle\right.
$$

and the system of Equation (1) reduces to

$$
\left\{\begin{array}{l}
\sigma_{1}=E\left(\varepsilon_{1}{ }^{K} t\right) \cdot \frac{\varepsilon_{1}{ }^{K} t+\varepsilon_{2}{ }^{K} t \cdot v\left(\varepsilon_{2}{ }^{K} t\right)}{1-v\left(\varepsilon_{1}{ }^{K} t\right) v\left(\varepsilon_{2}{ }^{K} t\right)}, \\
\sigma_{2}=E\left(\varepsilon_{2}{ }^{K} t\right) \cdot \frac{\varepsilon_{2}{ }^{K} t+\varepsilon_{1}{ }^{K} t \cdot v\left(\varepsilon_{1}{ }^{K} t\right)}{1-v\left(\varepsilon_{1}{ }^{K} t\right) v\left(\varepsilon_{2}{ }^{K} t\right)} .
\end{array}\right.
$$

The relation (6) simplifies to

$$
\begin{aligned}
W\left(\varepsilon_{1}{ }^{K}, \varepsilon_{2}{ }^{K}\right)=\int_{0}^{1} & {\left[E\left(\varepsilon_{1}{ }^{K} t\right) \frac{\varepsilon_{1}{ }^{K} t+\varepsilon_{2}{ }^{K} t \cdot v\left(\varepsilon_{2}{ }^{K} t\right)}{1-v\left(\varepsilon_{1}{ }^{K} t\right) v\left(\varepsilon_{2}{ }^{K} t\right)} \varepsilon_{1}{ }^{K}\right.} \\
& \left.+E\left(\varepsilon_{2}{ }^{K} t\right) \frac{\varepsilon_{2}{ }^{K} t+\varepsilon_{1}{ }^{K} t \cdot v\left(\varepsilon_{1}{ }^{K} t\right)}{1-v\left(\varepsilon_{1}{ }^{K} t\right) v\left(\varepsilon_{2}{ }^{K} t\right)} \varepsilon_{2}{ }^{K}\right] d t
\end{aligned}
$$

Let's take the function $E(\varepsilon)$ as known. The perturbation of functional (12) is possible due to functions $v\left(\varepsilon_{1}{ }^{K} t\right)$ and $v\left(\varepsilon_{2}{ }^{K} t\right)$. So on the base of assumptions (8) and (9) we have

$$
\begin{aligned}
\frac{\partial F}{\partial v\left(\varepsilon_{1}{ }^{K} t\right)}=0 \Leftrightarrow[ & \left.E\left(\varepsilon_{1}{ }^{K} t\right) \varepsilon_{1}{ }^{K} v\left(\varepsilon_{2}{ }^{K} t\right)+E\left(\varepsilon_{2}{ }^{K} t\right) \varepsilon_{2}{ }^{K}\right] \\
& \times \frac{\varepsilon_{1}{ }^{K} t+\varepsilon_{2}{ }^{K} t \cdot v\left(\varepsilon_{2}{ }^{K} t\right)}{\left[1-v\left(\varepsilon_{1}{ }^{K} t\right) v\left(\varepsilon_{2}{ }^{K} t\right)\right]^{2}}=0, \\
\frac{\partial F}{\partial v\left(\varepsilon_{1}{ }^{K} t\right)}=0 \Leftrightarrow & {\left[E\left(\varepsilon_{1}{ }^{K} t\right) \varepsilon_{1}{ }^{K}+E\left(\varepsilon_{2}{ }^{K} t\right) \varepsilon_{2}{ }^{K} v\left(\varepsilon_{1}{ }^{K} t\right)\right] } \\
\times & \frac{\varepsilon_{2}{ }^{K} t+\varepsilon_{1}{ }^{K} t \cdot v\left(\varepsilon_{1}{ }^{K} t\right)}{\left[1-v\left(\varepsilon_{1}{ }^{K} t\right) v\left(\varepsilon_{2}{ }^{K} t\right)\right]^{2}}=0,
\end{aligned}
$$

Because

$$
v\left(\varepsilon_{1}\right)=-\frac{\varepsilon_{2}}{\varepsilon_{1}} \text { and } v\left(\varepsilon_{2}\right)=-\frac{\varepsilon_{1}}{\varepsilon_{2}}
$$

the conditions (13) and (14) can be written as

$$
\frac{E\left(\varepsilon_{1}\right) v\left(\varepsilon_{2}\right)-E\left(\varepsilon_{2}\right) v\left(\varepsilon_{1}\right)}{1-v\left(\varepsilon_{1}\right) v\left(\varepsilon_{2}\right)}=0,
$$

or

$$
\frac{v\left(\varepsilon_{1}\right)}{v\left(\varepsilon_{2}\right)}=\frac{E\left(\varepsilon_{1}\right)}{E\left(\varepsilon_{2}\right)}
$$

For nonlinear materials the relations (15) are the definition of transversal strain coefficient, analogous to classical Poisson ratio definition in linear theory. The condition (17) between transversal strain and changeable stiffness coefficients is a coherence condition for material characteristics of nonlinear isotropic materials, as a consequence of the minimum energy principle.

\section{Conclusions}

Relations between material characteristics results from minimum energy principle. The strain energy density function can 
be treated as functional of materials characteristics. The solution of system of equations (8), (9) is not trivial in case if we know at least one characteristic.

\section{REFERENCES}

[1] R.W. Ogden, "Non-linear elastic deformations” Dover Publications, Mineola, New York 1997.

[2] P. Perzyna, "Coupling of dissipative mechanisms of viscoplastic flow“ Arch. Mechanics 29, 1977, pp. 607-624.

[3] P. Perzyna, "The thermodynamical theory of elasto-viscoplasticity“ Engineering Transactions 53, 2005, pp. 235-316.

[4] H. Petryk, "On the second-order work in plasticity" Archives of Mechanics, 37. Warszawa 1985, pp. 503-520.
[5] H. Petryk, "The energy criteria of instability in the time-independent inelastic solids" Archives of Mechanics, 43. 4. Warszawa 1991, pp. 519-545.

[6] H. Petryk, "On stability and symmetry conditions in time independent plasticity" Archives of Mechanics, 43. 2 -3. Warszawa 1991, pp. 377-397.

[7] J. Schroder, P. Neff, "Invariant Formulation of Hyperelastic Transverse Isotropy Based on Polyconvex Free Energy Functions” International Journal of Solids and Structures, Vol. 40, 2003, pp. 401-445.

[8] D.R. Wagner, J.C. Lotz, "A non-linear anisotropic strain energy function for the annulus fibrosus" San Francisco: Orthopaedic Research Society; 2001

[9] T. Wegner, D. Kurpisz "Phenomenological modeling of mechanical properties of metal foam” Journal of Theoretical and Applied Mechanics JTAM (in press). 